\title{
Many-Body Quantum Interference and the Saturation of Out-of-Time-Order Correlators
}

\author{
Josef Rammensee, Juan Diego Urbina, and Klaus Richter ${ }^{*}$ \\ Institut für Theoretische Physik, Universität Regensburg, D-93040 Regensburg, Germany
}

(Received 17 May 2018; published 20 September 2018)

\begin{abstract}
Out-of-time-order correlators (OTOCs) have been proposed as sensitive probes for chaos in interacting quantum systems. They exhibit a characteristic classical exponential growth, but saturate beyond the socalled scrambling or Ehrenfest time $\tau_{E}$ in the quantum correlated regime. Here we present a path-integral approach for the entire time evolution of OTOCs for bosonic $N$-particle systems. We first show how the growth of OTOCs up to $\tau_{E}=(1 / \lambda) \log N$ is related to the Lyapunov exponent $\lambda$ of the corresponding chaotic mean-field dynamics in the semiclassical large- $N$ limit. Beyond $\tau_{E}$, where simple mean-field approaches break down, we identify the underlying quantum mechanism responsible for the saturation. To this end we express OTOCs by coherent sums over contributions from different mean-field solutions and compute the dominant many-body interference term amongst them. Our method further applies to the complementary semiclassical limit $\hbar \rightarrow 0$ for fixed $N$, including quantum-chaotic single- and few-particle systems.
\end{abstract}

DOI: $10.1103 /$ PhysRevLett.121.124101

The study of signatures of unstable classical dynamics in the spectral and dynamical properties of corresponding quantum systems, known as quantum chaos [1], has recently received particular attention after the proposal of Kitaev [2] and related works [3-5] that address the mechanisms for spreading or "scrambling" quantum information across the many degrees of freedom of interacting many-body (MB) systems. With regard to such a MB quantum-to-classical correspondence, out-of-time-order correlators (OTOCs) [5,6], such as

$$
C(t)=\left\langle[\hat{V}(t), \hat{W}(0)]^{\dagger}[\hat{V}(t), \hat{W}(0)]\right\rangle,
$$

are measures of choice (with several experimental protocols already available [7-11]): The squared commutator of a suitable (local) operator $\hat{V}(t)$ with another (local) perturbation $\hat{W}(0)$ probes the temporal growth of $\hat{V}$, including its growing complexity. Hence, due to their unusual time ordering, OTOCs represent MB quantum analogues of classical measures for instability of chaotic MB dynamics. Indeed, invoking a heuristic classical-to-quantum correspondence for small $\hbar$ and replacing the commutator in Eq. (1) for short times by Poisson brackets one obtains, e.g., for $\hat{W}=\hat{p}_{i}, \hat{V}=\hat{q}_{j}[5,6,8]$,

$$
|i \hbar|^{2}\left\langle\left\{p_{i}^{(i)}, q_{j}^{(f)}(t)\right\}^{2}\right\rangle=\hbar^{2}\left\langle\left(\frac{\partial q_{j}^{(f)}}{\partial q_{i}^{(i)}}(t)\right)^{2}\right\rangle \propto \hbar^{2} e^{2 \lambda t} .
$$

Here the averages $\langle\cdots\rangle$ are taken over the initial phasespace points $(\mathbf{q}, \mathbf{p})$ weighted by the corresponding quasidistribution. The exponential growth on the rhs follows from the relation $\left|\partial q_{j}^{(f)} / \partial q_{i}^{(i)}\right| \propto e^{\lambda t}$ for chaotic systems with average single-particle (SP) Lyapunov exponent $\lambda$, see also Ref. [12] for another semiclassical derivation. Intriguingly, in view of Eq. (2), the genuinely quantummechanical OTOC $C(t)$ provides a direct measure of classical chaos in the corresponding quantum system, similar to the Loschmidt echo [13]. This close correspondence has been unambiguously observed in numerical studies for SP systems [14]. For MB problems analytical works have focused on Sachdev-Ye-Kitaev models $[15,16]$ or used random matrix theory (where $\lambda \rightarrow \infty$ ) [17-19], while the numerical identification of a MB Lyapunov exponent from Eq. (1) remains a challenge [20-22].

Moreover, Eq. (2) predicts unbounded classical growth while $C(t)$ is eventually bounded due to quantum mechanical unitarity. Indeed, $C(t)$ is numerically found $[14,20]$ to saturate beyond a characteristic time scale, known as Ehrenfest time $\tau_{E}[23,24]$ and dubbed scrambling time [5,25] in the MB context. $\tau_{E}$ separates initial quantum evolution following essentially classical motion from dynamics dominated by interference effects. Accordingly, quantum interference has been assumed to cause saturation of OTOCs in some way $[3,14,15,22]$, but to date the precise underlying dynamical mechanism has yet been unknown for chaotic SP and MB systems.

This classical-to-quantum crossover happens at $\tau_{E}=$ $(1 / \lambda) \log \left(1 / \hbar_{\text {eff }}\right)$ where " $\hbar_{\text {eff }} \rightarrow 0$ " can denote complementary semiclassical limits: For fixed $N, \hbar_{\text {eff }} \sim \hbar$ and $\lambda$ is the characteristic Lyapunov exponent of the limiting classical particle dynamics [see Eq. (2) for $N=1$ ]. For MB systems with a complementary classical, large- $N$ mean-field limit, $\hbar_{\mathrm{eff}} \simeq 1 / N$ and $\lambda$ characterizes the instability of the corresponding nonlinear mean-field solutions. 


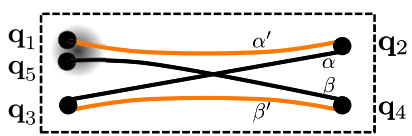

(a)

(c)

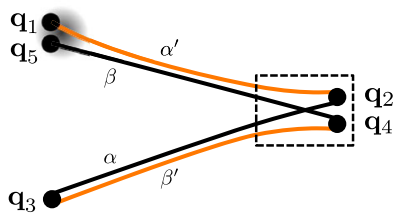

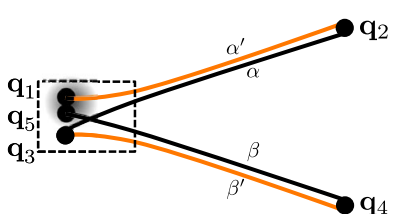

(b)

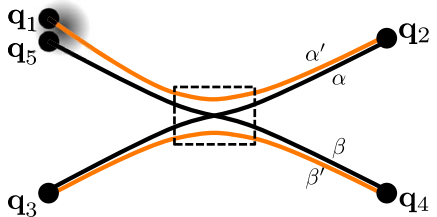

(d)
We take the expectation value for an initial wave packet $|\Psi\rangle$ localized in both quadratures (like a MB coherent state, generalizations are discussed later).

Our semiclassical method is based on approximating the path-integral representation of $\hat{U}(t)$ in Fock space by its asymptotic form for large $N$, the MB version [34] of the Van Vleck-Gutzwiller propagator [1],

$$
\begin{aligned}
K\left(\mathbf{q}^{(f)}, \mathbf{q}^{(i)} ; t\right) & =\left\langle\mathbf{q}^{(f)}|\hat{U}(t)| \mathbf{q}^{(i)}\right\rangle \\
& \simeq \sum_{\gamma: \mathbf{q}^{(i)} \rightarrow \mathbf{q}^{(f)}} A_{\gamma}\left(\mathbf{q}^{(f)}, \mathbf{q}^{(i)} ; t\right) e^{\left(i / \hbar_{\mathrm{eff}}\right) R_{\gamma}\left(\mathbf{q}^{(f)}, \mathbf{q}^{(i)} ; t\right)} .
\end{aligned}
$$

FIG. 1. Trajectory configurations representing interfering mean-field solutions that dominantly contribute to the OTOC $C(t)$, Eq. (7). The trajectory quadruples reside (a) inside an encounter (marked by dashed box), form a "two-leg" diagram with an encounter (b) at the beginning or (c) at the end, or (d) build a "four-leg" diagram with the encounter in between.

The notable interference-based saturation of OTOCs beyond $\tau_{E}$ is not captured by a Moyal expansion $[16,18]$ of commutators [such as Eq. (1)] in powers of $\hbar_{\mathrm{eff}}$ as implicit in Eq. (2). However, as originally developed for SP [26-33] and recently extended to MB systems [34-39], there exist semiclassical techniques that adequately describe post-Ehrenfest quantum phenomena. By extending these approaches to $\mathrm{MB}$ commutator norms, here we develop a unifying semiclassical theory for OTOCs which bridges classical mean-field and quantum MB concepts for bosonic large- $N$ systems. The complementary limit " $\hbar \rightarrow 0$ " for fixed $N$ will be discussed at the end. We express OTOCs through semiclassical propagators in Fock space [34] leading to sums over amplitudes from unstable classical paths, i.e., mean-field solutions. By considering subtle classical correlations amongst them we identify and compute the dominant contributions involving correlated MB dynamics swapping forth and back between mean-field paths (see Fig. 1). They proof responsible for the initial exponential growth and the saturation of OTOCs.

Specifically, we consider Bose-Hubbard systems with $n$ sites describing $N$ interacting bosons with Hamiltonian

$$
\hat{H}=\sum_{i j=1}^{n} h_{i j} \hat{b}_{i}^{\dagger} \hat{b}_{j}+\frac{1}{N} \sum_{i j k l=1}^{n} V_{i j k l} \hat{b}_{i}^{\dagger} \hat{b}_{j}^{\dagger} \hat{b}_{k} \hat{b}_{l},
$$

where $\hat{b}_{i}^{\dagger}\left(\hat{b}_{i}\right)$ are creation (annihilation) operators at sites $i=1, \ldots, n$. The parameters $h_{i j}$ define on-site energies and hopping terms, and $V_{i j k l}$ denote interactions.

We evaluate the OTOC Eq. (1) for position and momentum quadrature operators [40] $\hat{q}_{i}=\left(\hat{b}_{i}+\hat{b}_{i}^{\dagger}\right) / \sqrt{2 N}$, $\hat{p}_{i}=\left(\hat{b}_{i}-\hat{b}_{i}^{\dagger}\right) /(\sqrt{2 N} i)$, related to occupation operators $\hat{n}_{i}$ through $\left(\hat{q}_{i}^{2}+\hat{p}_{i}^{2}\right) / 2=\hbar_{\text {eff }}\left(\hat{n}_{i}+1 / 2\right)$. Using the MB time evolution operator $\hat{U}(t)=\exp (-i \hat{H} t / \hbar)$ Eq. (1) reads

$$
C(t)=\left\langle\Psi\left|\left[\hat{p}_{i}, \hat{U}^{\dagger}(t) \hat{q}_{j} \hat{U}(t)\right]\left[\hat{U}^{\dagger}(t) \hat{q}_{j} \hat{U}(t), \hat{p}_{i}\right]\right| \Psi\right\rangle .
$$

The sum runs over all (mean-field) solutions $\gamma$ of the classical equations of motion $i \partial \boldsymbol{\Phi} / \partial t=\partial \mathcal{H}^{\mathrm{cl}} / \partial \boldsymbol{\Phi}^{*}$ of the classical Hamilton function that denotes the mean-field limit of $\hat{H}$, Eq. (3), for $\hbar_{\mathrm{eff}}=1 / N \ll 1$ :

$$
\mathcal{H}^{\mathrm{cl}}(\mathbf{q}, \mathbf{p})=\frac{1}{\hbar} \sum_{i j=1}^{n} h_{i j} \Phi_{i}^{*} \Phi_{j}+\frac{1}{\hbar} \sum_{i j k l=1}^{n} V_{i j k l} \Phi_{i}^{*} \Phi_{j}^{*} \Phi_{k} \Phi_{l} .
$$

The initial and final real parts of the complex fields $\boldsymbol{\Phi}=$ $(\mathbf{q}+i \mathbf{p}) / \sqrt{2}$ are fixed by $\mathbf{q}^{(i)}$ and $\mathbf{q}^{(f)}$, but not their imaginary parts, thus generally admitting many timedependent mean-field solutions or "trajectories" $\gamma$ that enter the coherent sum in Eq. (5) and are ultimately responsible for $\mathrm{MB}$ interference effects. In Eq. (5) the phases are given by classical actions $R_{\gamma}\left(\mathbf{q}^{(f)}, \mathbf{q}^{(i)} ; t\right)=$ $\int_{0}^{t} d t^{\prime}\left[\mathbf{p}_{\gamma}\left(t^{\prime}\right) \cdot \dot{\mathbf{q}}_{\gamma}\left(t^{\prime}\right)-\mathcal{H}^{\mathrm{cl}}\left(\mathbf{q}_{\gamma}\left(t^{\prime}\right), \mathbf{p}_{\gamma}\left(t^{\prime}\right)\right)\right]$ along $\gamma$ and the weights $A_{\gamma}$ reflect their classical stability [see Eq. (30) in the Supplemental Material [41]]. We assume that the mean-field limit exhibits uniformly hyperbolic, chaotic dynamics where the exponential growth has the same Lyapunov exponent $\lambda$ at any phase space point. Here, we do not address questions concerning light cone information spreading and nonchaotic behavior, e.g., due to (partial) integrability or MB localization. Inserting unit operators in the position quadrature representation into Eq. (4) and using Eq. (5) for $K$ we get a general semiclassical representation of the OTOC. To leading order in $\hbar_{\text {eff }}$, derivatives $\hat{p}_{i}=-i \hbar_{\text {eff }} \partial / \partial q_{i}$ only act on the phases in $K$ and thus, using the relations $\partial R_{\gamma} / \partial \mathbf{q}^{(i)}=-\mathbf{p}_{\gamma}^{(i)}$, we obtain for the OTOC Eq. (4)

$$
\begin{aligned}
C(t) \simeq & \int d^{n} q_{1} \int d^{n} q_{2} \int d^{n} q_{3} \int d^{n} q_{4} \int d^{n} q_{5} \Psi^{*}\left(\mathbf{q}_{1}\right) \Psi\left(\mathbf{q}_{5}\right) \\
& \times \sum_{\substack{\alpha^{\prime}: \mathbf{q}_{1} \rightarrow \mathbf{q}_{2} \\
\alpha: \mathbf{q}_{3} \rightarrow \mathbf{q}_{2}}} A_{\alpha^{\prime}}^{*} A_{\alpha} e^{\left(i / \hbar_{\mathrm{eff}}\right)\left(-R_{\alpha^{\prime}}+R_{\alpha}\right)}\left(p_{\alpha^{\prime}, i}^{(i)}-p_{\alpha, i}^{(i)}\right) q_{\alpha, j}^{(f)} \\
& \times \sum_{\substack{\beta^{\prime}: \\
\beta: \mathbf{q}_{3} \rightarrow \mathbf{q}_{4} \\
\beta: \mathbf{q}_{5} \rightarrow \mathbf{q}_{4}}} A_{\beta^{\prime}}^{*} A_{\beta} e^{\left(i / \hbar_{\mathrm{eff}}\right)\left(-R_{\beta^{\prime}}+R_{\beta}\right)}\left(p_{\beta, i}^{(i)}-p_{\beta^{\prime}, i}^{(i)}\right) q_{\beta, j}^{(f)}
\end{aligned}
$$

The four time evolution operators in Eq. (4) have been transformed to fourfold sums over contributions from 
trajectories of temporal length $t$ linking different initial and final position quadratures. A schematic illustration of a representative trajectories quadruple that displays the geometric connections at the corresponding position quadratures $\mathbf{q}_{l}, l=1, \ldots, 5$, is given by

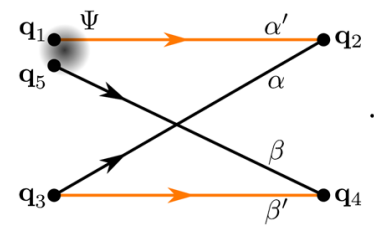

Black (orange) arrows refer to contributions to $K\left(K^{*}\right)$, and the gray shaded spot mimics the (localized) state $|\Psi\rangle$. The semiclassical approximation in Eq. (7) amounts to substitute $\hat{p}_{i}, \hat{q}_{j}$ in Eq. (4) by their classical counterparts $p_{\gamma, i}^{(i)}$ and $q_{\gamma, j}^{(f)}$ for $\gamma \in\left\{\alpha, \beta, \alpha^{\prime}, \beta^{\prime}\right\}$. The commutators themselves translate into differences of initial momenta of trajectories not restricted to start at nearby positions.

Since $R_{\gamma}\left(\mathbf{q}^{(f)}, \mathbf{q}^{(i)} ; t\right) \gg \hbar_{\text {eff }}$ in the semiclassical limit, the phase factors in Eq. (7) are generally highly oscillatory when integrating over initial or final positions. Hence, contributions from arbitrary trajectory quadruples are suppressed, while correlated quadruples with action differences such that $R_{\alpha}-R_{\alpha^{\prime}}+R_{\beta}-R_{\beta^{\prime}} \simeq \mathcal{O}\left(\hbar_{\text {eff }}\right)$ will dominantly contribute to $C(t)$. These are constellations where most of the time trajectories are pairwise nearly identical, except in so-called encounter regions in phase space where trajectory pairs approach each other, follow a correlated evolution, and exchange their partners.

For OTOCs the relevant quadruples involve a single encounter and can be subdivided into four classes depicted in Fig. 1: Diagram (a) represents a bundle of four trajectories staying in close vicinity to each other, i.e., forming an encounter, during the whole time $t$. Panels (b) and (c) display "two-leg" diagrams with an encounter at the beginning or end, and with uncorrelated dynamics of the two trajectory pairs ("legs") outside the encounter. The "four-leg" diagrams in (d) are characterized by uncorrelated motion before and after the encounter. The structure of the OTOC implies that the two legs on the same side of an encounter are of equal length.

Inside an encounter (boxes in Fig. 1) the hyperbolic dynamics essentially follows a common mean-field solution, i.e., linearization around one reference trajectory allows for expressing the remaining three trajectories. If their action differences are of order $\hbar_{\text {eff }}$ the time scale related to an encounter just corresponds to $\tau_{E}$ [Eqs. (20), (21) and (48) in Ref. [41]]. Because of the exponential growth of distances in chaotic phase space the dynamics merges at the encounter boundary into uncorrelated time evolution of two trajectory legs [see, e.g., trajectories $\alpha$ and $\beta$ in Fig. 1(b)]. Notably, Hamilton dynamics implies that this exponential separation along unstable manifolds in phase space is complemented by motion near stable manifolds, leading to the formation of (pairs of) exponentially close trajectories [29]. This mechanism gets quantum mechanically relevant for times beyond $\tau_{E}$ [see, e.g., paths $\alpha^{\prime}$ and $\alpha$ or $\beta$ and $\beta^{\prime}$ in Figs. 1(b) and (d)] and will prove crucial for semiclassically restoring unitarity and for explaining OTOC saturation.

The evaluation of Eq. (7) requires a thorough consideration of the dynamics in and around the encounter regions and the calculation of corresponding encounter integrals based on statistical averages invoking ergodic properties of chaotic systems. The detailed evaluation of the diagrams (a) to (d) in Fig. 1 as a function of $\tau_{E}$ for $\hbar_{\text {eff }} \ll 1$ is provided in Supplemental Material [41]. The $\tau_{E}$ dependence of related objects has been considered for a variety of spectral, scattering, and transport properties of chaotic SP systems [31-33,49-51]. Conceptually, our derivation follows along the lines of these works [52], but requires the generalization to high-dimensional MB phase space. Moreover, the encounter integrals involve additional amplitudes related to the operators in the OTOC that demand special treatment, depending on whether the initial or final position quadratures are inside an encounter.

Using furthermore the $A_{\gamma}$ in Eq. (7) to convert integrations over final positions into initial momenta, the OTOC contribution from each diagram is conveniently represented as phase-space average

$$
C(t) \simeq \int d^{n} q \int d^{n} p W(\mathbf{q}, \mathbf{p}) I(\mathbf{q}, \mathbf{p} ; t) .
$$

Here, $W(\mathbf{q}, \mathbf{p})=\int d^{n} y /\left(2 \pi \hbar_{\mathrm{eff}}\right)^{n} \Psi^{*}(\mathbf{q}+\mathbf{y} / 2) \Psi(\mathbf{q}-\mathbf{y} / 2)$ $\exp \left[\left(i / \hbar_{\mathrm{eff}}\right) \mathbf{y p}\right]$ is the Wigner function [55] of the initial state $\Psi$, and $I(\mathbf{q}, \mathbf{p} ; t)$ comprises all encounter integrals. As shown in Ref. [41] and sketched in Fig. 2, for times $t<\tau_{E}$ the only non-negligible contribution $I_{<}$originates from diagram (a), whereas a combination of diagrams (c) and (d) yields the contribution $I_{>}$nonvanishing for $t>\tau_{E}$.

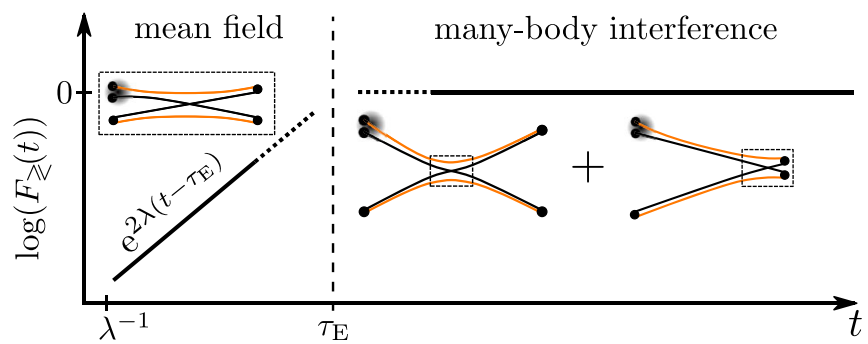

FIG. 2. Universal contributions to the time evolution of the OTOC $C(t)$ for classically chaotic many-body quantum systems before $\left[F_{<}(t)\right.$, Eq. (14)] and after $\left[F_{>}(t)\right.$, Eq. (16)] the Ehrenfest time $\tau_{E}=(1 / \lambda) \log (N)$ marked by the vertical dashed line. The insets show diagrams (a), (d), and (c) from Fig. 1, representing interfering mean-field solutions. Not shown is the crossover regime at $t \approx \tau_{E}$ to which all diagrams from Fig. 1 contribute. 
Using $\mathbf{x}^{(f)}(\mathbf{x} ; t)$ as the final phase space point of a trajectory originating from $\mathbf{x}=(\mathbf{q}, \mathbf{p})$, these terms read

$$
\begin{aligned}
& I_{<}(\mathbf{x} ; t)=F_{<}(t)\left(\sum_{l=1}^{n-2}\left[\mathbf{e}_{s}^{(l)}(\mathbf{x})\right]_{p_{i}}\left[\mathbf{e}_{u}^{(l)}\left(\mathbf{x}^{(f)}(\mathbf{x} ; t)\right)\right]_{q_{j}}\right)^{2}, \\
& I_{>}(\mathbf{x} ; t)=F_{>}(t)\left\langle\left(p_{i}-p_{i}^{\prime}\right)^{2}\right\rangle_{\mathbf{x}}\left(\left\langle q_{j}^{\prime 2}\right\rangle_{\mathbf{x}}-\left\langle q_{j}^{\prime}\right\rangle_{\mathbf{x}}^{2}\right) .
\end{aligned}
$$

Here $\left\langle f\left(\mathbf{x}^{\prime}\right)\right\rangle_{\mathbf{x}}$ denotes the average of a phase-space function $f$ over the manifold defined through $\mathbf{x}$ by constant energy and particle density [Eq. (35) in Ref. [41]]. In Eq. (10) the vectors $\mathbf{e}_{s / u}^{(l)}(\mathbf{x})$ denote the $n-2$ directions towards the stable, respectively unstable manifolds at $\mathbf{x}$, and the labels $q_{j}, p_{i}$ indicate components of those. Finally, in Eqs. (10), (11)

$$
\begin{aligned}
F_{<}(t)= & e^{2 \lambda\left(t-\tau_{E}\right)}\left(\frac{2}{\pi}\right)^{n-2}\left[\operatorname{Si}\left(e^{\lambda\left(\tau_{E}-t\right)}\right)\right]^{n-4} \\
& \times\left[\operatorname{Si}\left(e^{\lambda\left(\tau_{E}-t\right)}\right)-\sin \left(e^{\lambda\left(\tau_{E}-t\right)}\right)\right]^{2}, \\
F_{>}(t)= & {\left[\frac{2}{\pi} \operatorname{Si}\left(e^{\lambda \tau_{E}}\right)\right]^{n-2}-\left[\frac{2}{\pi} \operatorname{Si}\left(e^{\lambda\left(\tau_{E}-t\right)}\right)\right]^{n-2} }
\end{aligned}
$$

with $\operatorname{Si}(z)=\int_{0}^{z}\left(\sin \left(z^{\prime}\right) / z^{\prime}\right) d z^{\prime}$. In the semiclassical limit follows $\lambda \tau_{E}=\log \left(1 / \hbar_{\mathrm{eff}}\right) \gg 1$ such that $F_{<}\left(t>\tau_{E}\right)$ is strongly suppressed (reflecting the vanishing phase space volume of quadruples of trajectories remaining close to each other over longer times) and can be expressed by a Heaviside step function,

$$
F_{<}(t) \approx e^{2 \lambda\left(t-\tau_{E}\right)} \Theta\left(\tau_{E}-t\right)=\hbar_{\mathrm{eff}}^{2} e^{2 \lambda t} \Theta\left(\tau_{E}-t\right) .
$$

As a result the contribution to $C(t)$ in Eq. (9), associated with $I_{<}$and $F_{<}(t)$, is responsible for the initial exponential growth $\exp \left[2 \lambda\left(t-\tau_{E}\right)\right]$ of the OTOC for $t<\tau_{E}$, as also depicted in Fig. 2. It reflects unstable mean-field behavior. Note that for $t>\lambda^{-1}$ (the ergodic time) [42]

$$
\frac{\partial q_{j}^{(f)}}{\partial q_{i}^{(i)}}(\mathbf{x} ; t) \approx \sum_{l=1}^{n-2}\left[\mathbf{e}_{s}^{(l)}(\mathbf{x})\right]_{p_{i}}\left[\mathbf{e}_{u}^{(l)}\left(\mathbf{x}^{(f)}(\mathbf{x} ; t)\right)\right]_{q_{j}} e^{\lambda t},
$$

implying that our result, Eq. (10), reduces to the short-time limit, Eq. (2), of the commutator, but moreover additionally contains the missing cutoff through $\theta\left(\tau_{E}-t\right)$.

On the contrary, $F_{>}(t)$ in Eq. (13) is suppressed for $t<\tau_{E}$, but is indeed responsible for post-Ehrenfest OTOC saturation, as for $\lambda \tau_{E} \gg 1$ it can be approximated by

$$
F_{>}(t) \approx \Theta\left(t-\tau_{E}\right) .
$$

The underlying diagrams (c) and (d) represent dynamics swapping forth and back along distinctly different encounter-coupled mean-field solutions. This mechanism that emerges evidently in a regime where mean-field approaches fail [56] creates quantum correlations and entanglement, respectively [57]. The underlying MB interference, accounted for in the encounter integrals, is at the heart of $F_{>}(t)$ entering $I_{>}(\mathbf{x} ; t)$ in Eq. (11).

The latter further contains classical quantities that determine its saturation value: the variance of the $j$ th final position quadrature $\left(\Delta q_{j}^{\prime}\right)^{2}=\left\langle q_{j}^{\prime 2}\right\rangle_{\mathbf{x}}-\left\langle q_{j}^{\prime}\right\rangle_{\mathbf{x}}^{2}$ and $\left\langle\left(p_{i}-p_{i}^{\prime}\right)^{2}\right\rangle_{\mathbf{x}}$. A straightforward calculation of the ergodic averages, exploiting the connection between $p_{i}^{\prime 2}$ and $q_{j}^{\prime 2}$ with the particle density [see Eq. (18) in Ref. [41]] yields $I_{>}(\mathbf{x} ; t) \approx \theta\left(t-\tau_{E}\right)\left(p_{i}^{2}+1 / n\right) \times(1 / n)$.

For an initial state $|\Psi\rangle$ with a Wigner function sharply localized in phase space, the average Eq. (9) then gives

$$
C(t) \approx \frac{2}{n^{2}} \quad \text { for } t>\tau_{E},
$$

with corrections of $\mathcal{O}\left(\hbar_{\text {eff }}\right)$ due to the finite width. Interestingly, the same result, Eq. (17), holds if $|\Psi\rangle$ is an extended chaotic MB state with fixed energy and particle density.

We finally discuss several implications and conclusions:

(i) Generalization to OTOCs with other operators.-The entire line of reasoning can be generalized to OTOCs involving operators that are smooth functions of position and momentum quadratures for which a corresponding classical symbol exists [41].

(ii) Time-reversal (TR) invariance and higher-order quantum corrections.-Remarkably, the leading quantum correction [Fig. 1(d)] is of the same order as the classical mean-field contribution at $\tau_{E}$. Moreover, the absence of trajectory loops in the diagrams in Fig. 1, usually associated with weak localization-like corrections, implies that our results hold true for systems with and without TR symmetry. Diagrams involving more than one trajectory encounter generally yield further subleading contributions that can be susceptible to TR symmetry breaking. Their evaluation for OTOCs requires further research.

(iii) Small- $\hbar$ limit and SP systems.-Our semiclassical calculation of OTOCs in the large- $N$ limit can be readily generalized to systems of $N$ particles in $d$ spatial dimensions in the complementary limit of small $\hbar$, including the quantum chaotic SP case $N=1$. There, $\hbar_{\text {eff }}=\hbar / S \propto$ $\lambda_{\mathrm{dB}} / L$ where $\lambda_{\mathrm{dB}}$ is the de Broglie wavelength, and $S$ and $L$ are typical actions and length scales of the chaotic classical limit. Invoking the Gutzwiller propagator [1] in $n=d \cdot N$ dimensions in Eq. (5) the exponential increase of the OTOC $C_{N}(t)$ is then determined by the leading Lyapunov exponent $\lambda_{N}$ of the corresponding classical $N$-particle system (see, e.g., Refs. [12,14] for $N=1$ ). Our derivation shows that saturation sets in at the corresponding Ehrenfest time $\tau_{E}^{(N)} \sim\left(1 / \lambda_{N}\right) \log \left(\hbar_{\text {eff }}^{-1}\right)$. We can again evaluate $C_{N}(t)$ for $t>\tau_{E}^{(N)}$. For example, for chaotic billiards $\left\langle\left(p_{i}-p_{i}^{\prime}\right)^{2}\right\rangle=p_{i}^{2}+p^{2} / n$. Since $L$ corresponds to the 
overall system size $\mathcal{L}, \quad\left(\Delta q_{j}^{\prime}\right)^{2} \propto(\mathcal{L})^{2}=L^{2}$. Thus $C_{N}(t) \propto S^{2} / n$, where the typical action $S=\hbar / \hbar_{\text {eff }}$ arises here since $\left[\hat{q}_{j}, \hat{p}_{i}\right]=i \delta_{i j} \hbar=i \delta_{i j} S \hbar_{\mathrm{eff}}$. Within this line of reasoning, one can view Ref. [14] as a quantitative numerical confirmation of our semiclassical findings.

Interestingly, for many systems we can have $L \ll \mathcal{L}$, such as for the famous Lorentz gas [42]. It is composed of scattering disks or spheres for $d=2$ or 3 [59] with diameters setting the scale $L$. Then the dynamics is hyperbolic up to $\tau_{E}{ }^{(1)}$ before it becomes diffusive. This implies that $\left(\Delta q_{j}^{\prime}\right)^{2}$ in Eq. (11) scales linearly with time, $\left(\Delta q_{j}^{\prime}\right)^{2} \sim D t$, with diffusion constant $D$. Thus, beyond $\tau_{E}^{(1)}$ we expect $C_{1}(t)$ to first linearly increase before it saturates at the ergodic (Thouless) time $\mathcal{L}^{2} / D$. In SP systems with diffusive dynamics arising from quantum scattering at impurities, the transport time $t_{\text {tr }}$ takes the role of $\tau_{E}^{(1)}$. This implies a sharp increase of $C_{1}(t)$ for $t<t_{\text {tr }}$, as already predicted in Ref. [6], followed by the diffusive behavior discussed above.

(iv) Nonergodic many-body dynamics. - The nonlinear mean-field dynamics associated with the classical limit of MB Fock space is much less understood $[39,61,62]$ than its SP counterpart. If the MB dynamics is diffusive for $t>\tau_{E}$, we expect a similar time dependence for $C(t)$ as discussed in (iii). The propagator Eq. (5) is not restricted to chaotic dynamics, but also allows for investigating the imprint of more complex, e.g., mixed regular-chaotic, phase space dynamics on OTOCs or, more generally, on the stability of MB quantum evolution per se.

To conclude, we considered the time evolution of OTOCs by developing a general semiclassical approach for interacting large- $N$ systems. It links chaotic motion in the classical mean-field limit to the correlated quantum many-body dynamics in terms of interference between mean-field solutions giving rise to scrambling and entanglement. We uncovered the relevant many-body quantum interference mechanism that is responsible for the commonly observed saturation of OTOCs at the scrambling or Ehrenfest time. While we explicitly derived OTOCs for bosonic systems, similar considerations should be possible for fermionic many-body systems [63] posing an interesting problem for future research.

We thank T. Engl, B. Geiger, S. Tomsovic, D. Ullmo, and D. Waltner for helpful conversations. We acknowledge funding from Deutsche Forschungsgemeinschaft through Project No. Ri681/14-1.

*klaus.richter@physik.uni-regensburg.de

[1] M. C. Gutzwiller, Chaos in Classical and Quantum Mechanics (Springer, New York, 1991).

[2] A. Kitaev, Hidden Correlations in the Hawking Radiation and Thermal Noise, talk at Breakthrough Physics Prize Symposium, 2014, https://www.youtube.com/watch? $\mathrm{v}=\mathrm{OQ} 9 \mathrm{qN} 8 \mathrm{j} 7 \mathrm{EZI}$.
[3] Y. Sekino and L. Susskind, J. High Energy Phys. 10 (2008) 65.

[4] S. H. Shenker and D. Stanford, J. High Energy Phys. 03 (2014) 67.

[5] J. Maldacena, S. H. Shenker, and D. Stanford, J. High Energy Phys. 08 (2016) 106.

[6] A. I. Larkin and Y. N. Ovchinnikov, Sov. Phys. JETP 28, 1200 (1969).

[7] G. Zhu, M. Hafezi, and T. Grover, Phys. Rev. A 94, 062329 (2016).

[8] B. Swingle, G. Bentsen, M. Schleier-Smith, and P. Hayden, Phys. Rev. A 94, 040302 (2016).

[9] M. Campisi and J. Goold, Phys. Rev. E 95, 062127 (2017).

[10] J. Li, R. Fan, H. Wang, B. Ye, B. Zeng, H. Zhai, X. Peng, and J. Du, Phys. Rev. X 7, 031011 (2017).

[11] M. Gärttner, J. G. Bohnet, A. Safavi-Naini, M. L. Wall, J. J. Bollinger, and A. M. Rey, Nat. Phys. 13, 781 (2017).

[12] J. Kurchan, J. Stat. Phys. 171, 965 (2018).

[13] R. A. Jalabert and H. M. Pastawski, Phys. Rev. Lett. 86, 2490 (2001).

[14] E. B. Rozenbaum, S. Ganeshan, and V. Galitski, Phys. Rev. Lett. 118, 086801 (2017).

[15] D. Bagrets, A. Altland, and A. Kamenev, Nucl. Phys. B921, 727 (2017).

[16] T. Scaffidi and E. Altman, arXiv:1711.04768.

[17] E. J. Torres-Herrera, A. M. García-García, and L. F. Santos, Phys. Rev. B 97, 060303 (2018).

[18] J. Cotler, N. Hunter-Jones, J. Liu, and B. Yoshida, J. High Energy Phys. 11 (2017) 48.

[19] A. del Campo, J. Molina-Vilaplana, and J. Sonner, Phys. Rev. D 95, 126008 (2017).

[20] A. Bohrdt, C. B. Mendl, M. Endres, and M. Knap, New J. Phys. 19, 063001 (2017).

[21] H. Shen, P. Zhang, R. Fan, and H. Zhai, Phys. Rev. B 96, 054503 (2017).

[22] K. Hashimoto, K. Murata, and R. Yoshii, J. High Energy Phys. 10 (2017) 138.

[23] P. Ehrenfest, Zeit. für Phys. 45, 455 (1927).

[24] G. P. Berman and G. M. Zaslavsky, Physica (Amsterdam) 91A, 450 (1978).

[25] G. Dvali, D. Flassig, C. Gomez, A. Pritzel, and N. Wintergerst, Phys. Rev. D 88, 124041 (2013).

[26] S. Tomsovic and E. J. Heller, Phys. Rev. Lett. 67, 664 (1991).

[27] I. L. Aleiner and A. I. Larkin, Phys. Rev. B 54, 14423 (1996).

[28] O. Agam, I. Aleiner, and A. Larkin, Phys. Rev. Lett. 85, 3153 (2000).

[29] M. Sieber and K. Richter, Physica Scripta T90, 128 (2001).

[30] S. Müller, S. Heusler, P. Braun, F. Haake, and A. Altland, Phys. Rev. E 72, 046207 (2005).

[31] P. W. Brouwer and S. Rahav, Phys. Rev. B 74, 075322 (2006).

[32] P. Jacquod and R. S. Whitney, Phys. Rev. B 73, 195115 (2006).

[33] D. Waltner, M. Gutiérrez, A. Goussev, and K. Richter, Phys. Rev. Lett. 101, 174101 (2008).

[34] T. Engl, J. Dujardin, A. Argüelles, P. Schlagheck, K. Richter, and J. D. Urbina, Phys. Rev. Lett. 112, 140403 (2014). 
[35] J. D. Urbina, J. Kuipers, S. Matsumoto, Q. Hummel, and K. Richter, Phys. Rev. Lett. 116, 100401 (2016).

[36] T. Engl, J. D. Urbina, and K. Richter, Phys. Rev. E 92, 062907 (2015).

[37] R. Dubertrand and S. Müller, New J. Phys. 18, 033009 (2016).

[38] M. Akila, D. Waltner, B. Gutkin, P. Braun, and T. Guhr, Phys. Rev. Lett. 118, 164101 (2017).

[39] S. Tomsovic, P. Schlagheck, D. Ullmo, J. D. Urbina, and K. Richter, Phys. Rev. A 97, 061606 (2018).

[40] G.S. Agarwal, Quantum Optics (Cambridge University Press, Cambridge, England, 2013).

[41] See Supplemental Material at http://link.aps.org/ supplemental/10.1103/PhysRevLett.121.124101 for the detailed technical evaluation of the diagrams (a) to (d) in Fig. 1 and the technical discussion of the generalization to other operators. It cites Refs. [30,31,33,42-48].

[42] P. Gaspard, Chaos, Scattering and Statistical Mechanics (Cambridge University Press, Cambridge, England, 1998).

[43] S. Müller, S. Heusler, P. Braun, and F. Haake, New J. Phys. 9, 12 (2007).

[44] M. Turek and K. Richter, J. Phys. A 36, L455 (2003).

[45] D. Spehner, J. Phys. A 36, 7269 (2003).

[46] D. Waltner, Semiclassical Approach to Mesoscopic Systems: Classical Trajectory Correlations and Wave Interference (Springer, Berlin, Heidelberg, 2012).

[47] P. W. Brouwer and S. Rahav, Phys. Rev. B 74, 085313 (2006).

[48] W. Schleich, Quantum Optics in Phase Space (Wiley-VCH, Weinheim, Germany, 2001).

[49] I. Adagideli, Phys. Rev. B 68, 233308 (2003).

[50] P. W. Brouwer, Phys. Rev. B 76, 165313 (2007).

[51] J. Kuipers, D. Waltner, C. Petitjean, G. Berkolaiko, and K. Richter, Phys. Rev. Lett. 104, 027001 (2010).
[52] Specific diagrams similar to class (d) in Fig. 1 have been considered in the context of shot noise $[50,53,54]$ and quantum chaotic SP [51] and MB [35] scattering.

[53] A. Lassl, Semiklassik jenseits der Diagonalnäherung: Anwendung auf ballisitische Systeme, Diploma thesis, Universität Regensburg, 2003.

[54] P. Braun, S. Heusler, S. Müller, and F. Haake, J. Phys. A 39, L159 (2006).

[55] A. M. Ozorio de Almeida, Hamiltonian Systems: Chaos and Quantization (Cambridge University Press, Cambridge, England, 1990).

[56] X. Han and B. Wu, Phys. Rev. A 93, 023621 (2016).

[57] It may be viewed as the underlying dynamical mechanism, supporting (in the large- $N$ limit) models for OTOCs based on coupled binaries [58].

[58] T. Rakovszky, F. Pollmann, and C. W. von Keyserlingk, arXiv:1710.09827 [Phys. Rev. X (to be published)].

[59] Ehrenfest time effects in Lorentz gases were studied, e.g., in Refs. [27,50,60].

[60] O. Yevtushenko, G. Lütjering, D. Weiss, and K. Richter, Phys. Rev. Lett. 84, 542 (2000).

[61] F. Borgonovi, F. M. Izrailev, and L. F. Santos, arXiv: 1802.08265

[62] S. Tomsovic, Phys. Rev. E 98, 023301 (2018).

[63] To this end, the semiclassical (large- $N$ ) approximation for the microscopic path integral propagator of discrete fermionic quantum fields [64] can be employed. Based on this fermionic propagator, a semiclassical calculation of a $\mathrm{MB}$ spin echo gave perfect agreement with numerical quantum calculations, see Ref. [65].

[64] T. Engl, P. Plöß1, J. D. Urbina, and K. Richter, Theor. Chem. Acc. 133, 1563 (2014).

[65] T. Engl, J. D. Urbina, K. Richter, and P. Schlagheck, Phys. Rev. A 98, 013630 (2018). 